Pacific Journal of Mathematics

UNIFORM APPROXIMATION BY POLYNOMIALS WITH 


\title{
UNIFORM APPROXIMATION BY POLYNOMIAIS WITH INTEGRAL COEFFICIENTS I
}

\author{
Le Baron O. Ferguson
}

\begin{abstract}
Throughout this paper $A$ will denote a discrete subring of the complex number plane $C$ with rank 2. For example, $A$ could be the Gaussian integers $Z+i Z$, where $Z$ denotes the rational integers, or the ring of integers of any imaginary quadratic field. We are concerned with characterizing those functions defined on a compact subset $X$ of $C$ which can be uniformly approximated by polynomials with coefficients in $A$. We say that such functions are $A$-approximable on $X$. We also consider the real case where $X$ is any compact subset of the reals $R$ and the coefficients of the approximating polynomials lie in $Z$ or any discrete subring of $R$. The real case is completely solved in the sense that a necessary and sufficient condition in order that a function can be so approximated is found. The complex case is solved if, in addition to being compact, $X$ either has transfinite diameter at least unity or void interior and connected complement.
\end{abstract}

The case where $X$ has transfinite diameter less than unity and nonvoid interior will be the subject of a later paper.

The complex case was solved by Fekete when the ring of coefficients $A$ is the ring of integers of an imaginary quadratic field. His results were announced in [4], but, as far as we know, proofs were never published. In [4] is found the key notion of the "algebraic kernel" of a compact subset of $C$ with respect to an imaginary quadratic field. This notion is extended here so as to be relevant to any ring $A$ defined above and a second characterization of the algebraic kernal (herein denoted by $J_{0}(X, A)$ ) is found. The set $J_{0}(X, A)$ seems to be difficult to determine, in general. Its calculation will be the subject of a future paper.

The real case was solved for intervals in Hewitt and Zuckerman [7]. We use the results obtained here in the complex case to extend their results to arbitrary compact subsets of $\boldsymbol{R}$.

Throughout this paper we endeavor to follow the terminology and notation in [2]. We use the symbol $C(X)$ to denote the set of all complex-valued continuous functions defined on $X$ and $C^{r}(X)$ to denote the real valued members of $C(X)$. If $f \in C(X)$ and $S \subset X$ we define $\|f\|_{S}=\sup \{|f(x)|: x \in S\}$. We frequently write $\|f\|$ for $\|f\|_{X}$.

2. Discrete rings and imaginary quadratic fields. We mention here the nonstandard results on these rings which will be needed 
repeatedly.

If $F$ is a field extension of $\boldsymbol{Q}$ we denote the ring of algebraic integers of $F$ by $I_{F}$. If $L$ is an imaginary quadratic field it is easy to see that $I_{L}$ is a discrete subring of $C$ and has rank 2 . On the other hand every ring $A$ with these properties is contained in $I_{L}$ for some imaginary quadratic field $L[11$, p. 150, Nr. 203]. Since $A$ has rank $2, L$ is uniquely determined by the inclusion $A \subset I_{L}$. Using the well known explicit representations for the elements of $I_{L}[13, \mathrm{p} .234]$ it is easy to see that there exists a positive integer $m$ such that $m I_{L} \subset A$.

Using the fact that $A$ has rank 2 and Theorem 1 of [3, p. 77] it is not hard to see that there exists $\delta>0$, depending only on $A$, such that if $z \in C$, there exists $a \in A$ with $|z-a|<\delta$.

3. Chebyshev polynomials and transfinite diameter. Let $X$ be a compact subset of $C$ and $n$ a positive integer. If $X$ is infinite the $n^{\text {th }}$ Chebyshev polynomial $t_{n}(z, X)$ for $X$ is defined to be the unique monic polynomial of degree $n$ such that

$$
\left\|t_{n}(z, X)\right\|_{X}=\inf \|t\|_{X}
$$

where the inf is taken over all such polynomials. If $X$ contains $m$ elements, where $m$ is finite, then we define $t_{n}(z, X)$ as above for $n \leqq m$ and set $t_{n}(z, X)=\Pi_{x \in X}(z-x)$ for $n>m$.

The existence of $t_{n}(z, X)$ is a direct consequence of $[1, \mathrm{p} .10]$. The uniqueness follows from [12, p. 36, Th. 1].

We define the transfinite diameter $d(X)$ of $X$ by

$$
d(X)=\lim _{n \rightarrow \infty}\left\|t_{n}(z, X)\right\|^{1 / n}
$$

(See [8, p. 226, Th. 16.1.2].)

The importance for us of the concept of transfinite diameter is contained in the following.

Proposition 3.1. Let $X$ be a compact subset of $C$ with $d(X) \geqq 1$. Then a complex valued function $f$ on $X$ is $A$-approximable on $X$ if and only if it is already an element of $A[z]$.

Proof. Suppose that $f$ is $A$-approximable on $X$ but $f \notin A[z]$. Then there exist $p_{1}$ and $p_{2}$ in $A[z]$ such that $p_{1} \neq p_{2}$ and $\left\|p_{i}-f\right\|<1 / 2$ for $i=1,2$. Thus $\left\|p_{1}-p_{2}\right\|<1$. Since $A$ is discrete, the leading coefficient of $p_{1}-p_{2}$ has modulus at least one and dividing $p_{1}-p_{2}$ by this coefficient gives a monic polynomial $p$ with $\|p\|<1$. From the existence of such a $p$ it is easy to see that $d(X)<1$, a contradiction. 
4. The algebraic kernel. In this section we define the algebraic kernel of a compact subset $X$ of $C$ with respect to $A$. We then give a necessary condition in order that a function be $A$-approximable.

Definition 4.1. Let $R$ be any subring of $C$ and $f$ a complex valued function on a subset $X$ of $C$. We say that $f$ is $R$-matchable on a subset $S$ of $X$ if there exists $p \in R[z]$ such that $p(z)=f(z)$ for all $z \in S$.

Definition 4.2. If $R$ is any subring of $C$ and $X$ is a compact subset of $C$ we define

$$
B(X, R)=\left\{p \in R[z]:\|p\|_{X}<1\right\} .
$$

Note that in 3.1 we have proved something stronger than the proposition. In fact we see that if $R$ is a discrete subring of $C$ and $X$ is a compact subset of $C$ with $d(X) \geqq 1$, then $R[z]$ is a discrete and therefore closed subring of $C(X)$. Indeed, we can prove that $B(X, R)=\{0\}$ as follows. If $g \in B(X, R)$ and $g$ is not identically zero on $X$ then we can divide by its leading coefficient to obtain a monic polynominal $p$ such that $0<\|p\|_{X}<1$ and derive a contradiction to $d(X) \geqq 1$ as in the proof of 3.1. Now, by [6, p. 35, (5.10)] since $R[z]$ is a discrete (additive) subgroup of $C(X)$ it is closed in $C(X)$.

Definition 4.3. For any subring $R$ of $C$ and compact subset $X$ of $C$ we define

$$
J(X, R)=\{z \in X: p(z)=0 \text { for all } p \in B(X, R)\} .
$$

When no confusion is possible we write $J(X)$ or simply $J$ for $J(X, R)$. If $A$ is a discrete subring of $C$ with rank 2 then by $\S 2$ there exists exactly one imaginary quadratic field $L$ such that $A \subset I_{L}$. With this fact in mind we make the following definition.

Definition 4.4. Let $X$ be a compact subset of $C$ and $L$ the imaginary quadratic field such that $A \subset I_{L}$. We define $J_{0}(X, A)$ to be the union of the complete sets of conjugates integral over $I_{L}$ which are entirely contained in $X$.

We note that $J_{0}\left(X, I_{L}\right)$ is what Fekete called the "algebraic kernel" of $X$ with respect to the field $L$ [4, p. 1338].

Proposition 4.5. If $X$ is a compact subset of $C$, then

$$
J_{0}(X, A) \subset J(X, A) \text {. }
$$


Proof. Suppose $A \subset I_{L}$. Let $\theta$ be in $J_{0}(X, A)$ and $q \in A[z]$ with $\|q\|_{X}<1$. We can write the conjugates of $\theta$ over $L$ as $\sigma_{1}(\theta), \sigma_{2}(\theta)$, $\cdots, \sigma_{n}(\theta)$ where the $\sigma_{i}$ 's are automorphisms of the splitting field $F$ of $\theta$ which leave $L$ pointwise fixed. Since $J_{0}(X, A)$, hence $X$, contains the conjugates we have

$$
1>\left|\prod_{i=1}^{n} q\left(\sigma_{i}(\theta)\right)\right|=\left|\prod_{i=1}^{n} \sigma_{i}(q(\theta))\right|=\left|N_{L}^{F}(q(\theta))\right| .
$$

Because $q(\theta)$ is integral over $I_{L}$ we have $N_{L}^{F}(q(\theta)) \in I_{L}$. Since $I_{L}$ is discrete and $\left|N_{L}^{F}(q(\theta))\right|<1, N_{L}^{F}(q(\theta))=0$. Thus $q(\theta)=0$.

Proposition 4.6. If $X$ is a compact subset of $C$, then in order that a complex valued function $f$ be $A$-approximable on $X$, it is necessary that $f$ be $A$-matchable on $J(X, A)$.

Proof. Suppose that $f$ is $A$-approximable on $X$. That is, there is a sequence $\left(p_{n}\right)$ of polynomials in $A[z]$ which tends uniformly to $f$ on $X$. Then there is an integer $N$ such that $m, n>N$ implies $\left\|p_{n}-p_{m}\right\|<1$. Then $p_{n}-p_{m}$ is an element of $B(X, A)$ and so $p_{n}-p_{m}=0$ on $J(X, A)$. Thus $m>N$ implies that $p_{m}$ matches $f$ on $J(X, A)$.

5. The complex case. In this section we prove a necessary and sufficient condition for approximability over a class of compact subsets of $\boldsymbol{C}$ defined below.

Definition 5.1. A compact subset $X$ of $C$ is said to be Lavrent'ev if $C[z]$ is uniformly dense in $C(X)$.

This terminology stems from the fact that in 1934 Lavrent'ev proved the following [9].

Proposition 5.2. A compact subset $X$ of $C$ is Lavrent'ev if and only if it has void interior and connected complement.

Proposition 5.3. Let $A$ contain the identity and let $X$ be a compact subset of $C$. If there exists a monic polynomial $p \in C[z]$ with $\|p\|<1$, then there exists a monic polynomial $q \in A[z]$ with $\|q\|<1$.

Proof. Let $n$ be the degree of $p$. Then define a sequence (starting with the integer $n$ ) of monic polynomials as follows. For $m \geqq n$, set $m=k n+r$ where $0 \leqq r<n$ and $k \geqq 1$. Then let

$$
p_{m}(z)=z^{r} p(z)^{k} \text {. }
$$


Note that $p_{m}$ has degree $m$. Also, if $s=\|p\|<1$ set $t=s^{1 / n}(t \geqq 0)$ so that $s=t^{n}$; it is clear that $0 \leqq t<1$. Next pick a real number $M$ such that

$$
\left\|z^{i}\right\|<s M \text { for } 1 \leqq i \leqq n,
$$

if $s>0$, or set $M=0$ if $s=0$. Then writing $m$ as above we have

$$
\left\|p_{m}\right\| \leqq\|p\|^{k}\left\|z^{r}\right\| \leqq s^{k} s M=t^{n k+n} M \leqq t^{n k+r} M=t^{m} M .
$$

Now fix a positive integer $j \geqq n-1$ such that

$$
\delta M t^{j+1} /(1-t)<1 / 3,
$$

where $\delta>1$ satisfies the conditions in $\S 2$. For each $m>j$ we define a polynomial $q_{m}$ as follows. Set

$$
q_{m}=\alpha_{0} p_{m}+\alpha_{1} p_{m-1}+\cdots+\alpha_{m-j-1} p_{j+1}
$$

where the $\alpha$ 's are defined as follows. Let $\alpha_{0}=1$. Let $\beta$ be the coefficient of $z^{m-1}$ in $\alpha_{0} p_{m}$. By the definition of $\delta$, there is a $\beta^{\prime} \in A$ such that $\left|\beta^{\prime}-\beta\right|<\delta$. Then if we set $\alpha_{1}=\beta^{\prime}-\beta$, we have that $p^{\prime}=\alpha_{0} p_{m}+\alpha_{1} p_{m-1}$ has leading coefficient $\alpha_{0}=1$ since the degree of $\alpha_{1} p_{m-1}$ is less than that of $p_{m}$. Also, the coefficient of $z^{m-1}$ in $p^{\prime}$ is the element $\beta+\left(\beta^{\prime}-\beta\right)=\beta^{\prime}$ of $A$. Continuing in this way, we pick $\alpha$ 's such that $\left|\alpha_{i}\right|<\delta$ for $1 \leqq i \leqq m-j-1$ and the coefficients of $z^{m}, \cdots, z^{j+1}$ in $q_{m}$ are elements of $A$. We have

$$
\begin{aligned}
\left\|q_{m}\right\| & \leqq \sum_{i=0}^{m-j-1}\left\|\alpha_{i} p_{m-i}\right\| \leqq \sum_{i=0}^{m-j-1} \delta t^{m-i} M \\
& =\delta M t^{j+1} \sum_{i=0}^{m-j-1} t^{i} \leqq \delta M \frac{t^{j+1}}{1-t}<1 / 3 .
\end{aligned}
$$

Next, if $m>j$, we define the $(j+1)$-tuple

$$
\left(\left(a_{m 0}\right), \cdots,\left(a_{m j}\right)\right)
$$

as follows. If $a_{m i}$ is the coefficient of $z^{i}$ in $q_{m}(0 \leqq i \leqq j)$, then let $\left[a_{m i}\right]$ be an element of $A$ closest to $a_{m i}$ and set $\left(a_{m i}\right)=a_{m i}-\left[a_{m i}\right]$, so that $\left|\left(a_{m i}\right)\right|<\delta$. As $m$ varies, these $(j+1)$-tuples remain in the product space $(\delta D)^{j+1}$, where $D$ is the closed unit disk in $C$.

Now if

$$
M^{\prime}=\max \left\{\left\|z^{i}\right\|: 0 \leqq i \leqq j\right\},
$$

we choose $\varepsilon^{\prime}>0$ such that

$$
\varepsilon^{\prime}(j+1) M^{\prime}<1 / 3 .
$$

Then, since $(\delta D)^{j+1}$ is compact in the topology given by the norm 


$$
\text { || }\left(z_{0}, \cdots, z_{j}\right)\left|\|=\max _{0 \leqq i \leqq j}\right| z_{i} \mid,
$$

there exist distinct $m_{1}$ and $m_{2}$ such that

$$
\text { III }\left(\left(a_{m_{1} 0}\right), \cdots,\left(a_{m_{1} j}\right)\right)-\left(\left(a_{m_{2} 0}\right), \cdots,\left(a_{m_{2} j}\right)\right)|| \mid<\varepsilon^{\prime} .
$$

We then have

$$
\begin{aligned}
\sum_{i=0}^{j} \mid & \left(a_{m_{1} i}\right)-\left(a_{m_{2} i}\right) \mid\left\|z^{i}\right\| \\
& \leqq(j+1) \max _{0 \leqq i \leqq j}\left\{\left|\left(a_{m_{1} i}\right)-\left(a_{m_{2} i}\right)\right|\left\|z^{i}\right\|\right\} \\
& <(j+1) \varepsilon^{\prime} M^{\prime} \\
& <1 / 3 .
\end{aligned}
$$

We now combine these estimates as follows. From (3) we infer that

$$
\left\|q_{m_{1}}-q_{m_{2}}\right\| \leqq\left\|q_{m_{1}}\right\|+\left\|q_{m_{2}}\right\|<2 / 3 .
$$

If $q_{m}^{\prime}$ denotes $q_{m}$ with $\left[a_{m_{i}}\right]$ in place of $a_{m_{i}}$ for $0 \leqq i \leqq j$, then (4) shows that

$$
\begin{aligned}
\|\left(q_{m_{1}}-q_{m_{2}}\right) & -\left(q_{m_{1}}^{\prime}-q_{m_{2}}^{\prime}\right) \| \\
& =\left\|\left(q_{m_{1}}-q_{m_{1}}^{\prime}\right)-\left(q_{m_{2}}-q_{m_{2}}^{\prime}\right)\right\| \\
& \leqq \sum_{i=0}^{j}\left|\left(a_{m_{1}}\right)-\left(a_{m_{2}}\right)\right|\left\|z^{i}\right\|<1 / 3 .
\end{aligned}
$$

Combining (5) and (6) we obtain

$$
\left\|q_{m_{1}}^{\prime}-q_{m_{2}}^{\prime}\right\|<1 \text {. }
$$

Also $q_{m_{1}}^{\prime}-q_{m_{2}}^{\prime}$ is a monic polynomial because each $q_{m}$ is monic with degree $m$ and $m_{1} \neq m_{2}$. Thus we can take $q=q_{m_{1}}^{\prime}-q_{m_{2}}^{\prime}$ in the proposition.

Corollary 5.4. If $A$ contains the identity and $X$ is a compact subset of $C$ with $d(X)<1$, then there is a monic polynomial $q \in A[z]$ with $\|q\|_{X}<1$.

Lemma 5.5. Let $q$ be a monic polynomial in $A[z]$ with $\|q\|_{X}<1$ and $b \in C[z]$. Then there exists [b] in $A[z]$ and $M$ not depending on $b$ such that

$$
\|b-[b]\|_{X}<M
$$

Proof. Since $n=\operatorname{deg} q$ is at least 1 we can write 


$$
b=b_{0}+b_{1} q+\cdots+b_{k} q^{k}
$$

where each $b_{i} \in C[z]$ and $\operatorname{deg} b_{i}<\operatorname{deg} q$ for $0 \leqq i \leqq k$. For each $i$ let $\left[b_{i}\right]$ be the polynomial obtained from $b_{i}$ by replacing each coefficient by a nearest element of $A$. Then with $\delta$ as in $\S 2$ we have

$$
\left\|b_{i}-\left[b_{i}\right]\right\| \leqq \sum_{j=0}^{n-1}\left\|\delta z^{j}\right\|=M_{0} \quad 0 \leqq i \leqq k,
$$

where the last equality serves to define $M_{0}$. We have

$$
\begin{aligned}
\left\|b-\sum_{i=0}^{k}\left[b_{i}\right] q^{i}\right\| & =\sum_{i=0}^{k}\left(b_{i}-\left[b_{i}\right]\right) q^{i} \\
& \leqq \sum_{i=0}^{k}\left\|b_{i}-\left[b_{i}\right]\right\|\|q\|^{i} \\
& <M_{0} /(1-\|q\|) .
\end{aligned}
$$

Lemma 5.6. Let $X$ be a compact subset of $C$ and suppose further that

(i) $X$ is Lavrent'ev;

(ii) $f \in C(X)$;

(iii) $q$ is a monic polynomial in $A[z]$ with $\|q\|_{X}<1$;

(iv) for any $\varepsilon>0$ there is an $r \in A[z]$ such that $|f(z)-r(z)|<\varepsilon$ whenever $q(z)=0, z \in X$.

Then $f$ is A-approximable on $X$.

Proof. Let $Z_{q}$ be the set of roots of $q$ which lie in $X$. Let $\varepsilon$ be any positive number. By (iv) there is an $r \in A[z]$ such that

$$
|f(z)-r(z)|<\varepsilon / 4 \text { for } z \in Z_{q} \text {. }
$$

Then by continuity, there is, for each $\alpha \in Z_{q}$ a closed disk $D_{\alpha}$ with center $\alpha$ and radius $\rho_{\alpha}$ such that the family $\left\{D_{\alpha}\right\}_{\alpha \in Z_{q}}$ is pairwise disjoint and

$$
|f(z)-r(z)|<\varepsilon / 2 \text { for } z \in D_{\alpha} \cap X .
$$

Plainly there is a continuous function $u$ mapping $X$ into $[0,1]$ such that $u(z)=1$ for $z$ in no $D_{\alpha}$ and $u(z)=0$ if for some $\alpha \in Z_{q}, z$ is in the closed disk of radius $\rho_{\alpha} / 2$ and centered at $\alpha$. It is easy to see that

$$
\|u(f-r)-(f-r)\|<\varepsilon / 2 \text {. }
$$

By 5.5 there is a positive integer $N$ such that

$$
\left\|b q^{N}-[b] q^{N}\right\| \leqq \varepsilon / 4
$$

for every $b \in C[z]$. Now consider $u(f-r) / q^{N}$, which is defined to be zero whenever $q$ is zero. It is continuous by construction. Thus by 
(i), there is an element $b \in C[z]$ such that

$$
\left\|\frac{u(f-r)}{q^{N}}-b\right\|<\varepsilon / 4 \text {. }
$$

It follows that

$$
\left\|u(f-r)-b q^{N}\right\|<\|q\|^{N} \varepsilon / 4<\varepsilon / 4 .
$$

Then by (2)

$$
\left\|u(f-r)-[b] q^{N}\right\|<\varepsilon / 2
$$

and by (1)

$$
\left\|(f-r)-[b] q^{N}\right\|<\varepsilon
$$

or

$$
\left\|f-\left(r+[b] q^{N}\right)\right\|<\varepsilon .
$$

THEOREM 5.7. Let $X$ be a Lavrent'ev subset of $C$ with $d(X)<1$. If $f$ is a complex valued function on $X$ then the following are equivalent.

(i ) $f$ is A-approximable on $X$;

(ii) $f$ is continuous and $A$-matchable on $J_{0}(X, A)$.

Proof. From 4.6 and 4.5 we see that (i) implies (ii). To prove the converse first note that if $f=p$ on $J_{0}(X, A)$ and $p \in A[z]$, then it suffices to approximate $f-p$ which is zero on $J_{0}(X, A)$. Hence we assume that $f=0$ on $J_{0}(X, A)$. Let $L$ be the imaginary quadratic field such that $A \subset I_{L}$. By $\S 2$ there exists a positive integer $m$ such that $m I_{L} \subset A$. Thus if $p \in I_{L}[z]$ and $\|f / m-p\|<\varepsilon / m$ then $\|f-m p\|<\varepsilon$ and $m p \in A[z]$. In view of this we assume that $A=I_{L}$.

Then $A, X$, and $f$ satisfy the hypotheses of 5.6 and it only remains to show that 5.6 (iii) and (iv) hold. By 5.4 there exists a monic $q \in A[z]$ with $\|q\|_{X}<1$, so that 5.6 (iii) is satisfied. Let $Z_{q}$ denote the set of all zeroes of $q$ which lie in $X$. Write $J_{0}(X, A)$ as the union of the sets of zeroes of a set of monic irreducible polynomials, $\left\{q_{1}, \cdots, q_{s}\right\}$, in $I_{L}[z]$. Denote the remaining elements of $Z_{q}$ by $\alpha_{1}, \cdots, \alpha_{k}$ so that

$$
Z_{q}=J_{0}(X, A) \cup\left\{\alpha_{1}, \cdots, \alpha_{k}\right\} .
$$

By the definition of $J_{0}(X, A)$ the $\alpha_{i}$ 's form a set of algebraic numbers which does not contain a complete set of conjugates over $L$. In view of this 7.3 can be applied to give $p \in A[z]$ such that 


$$
\left|p\left(\alpha_{i}\right)-\frac{f\left(\alpha_{i}\right)}{q_{1} \cdots q_{s}\left(\alpha_{i}\right)}\right|<\frac{\varepsilon}{\left|q_{1} \cdots q_{s}\left(\alpha_{i}\right)\right|} \quad \text { for } 1 \leqq i \leqq k
$$

Then

$$
\left|p q_{1} \cdots q_{s}(z)-f(z)\right|<\varepsilon \text { for } z \in Z_{q}
$$

and $p q_{1} \cdots q_{s} \in A[z]$ which shows that 5.6 (iv) is satisfied.

TheOREM 5.8. Let $X$ be a Lavrent'ev subset of $C$ with $d(X)<1$. Then a continuous complex valued function $f$ on $X$ is A-approximable if and only if its Lagrange interpolating polynomial $r$ on $J_{0}(X, A)$ is an element of $A[z]$.

Proof. By 5.7 the condition $r \in A[z]$ is sufficient for the $A$-approximability of $f$ since $r$ matches $f$ on $J_{0}(X, A)$. Conversely, from 5.7 we know that if $f$ is $A$-approximable then there is a $p \in A[z]$ which matches $f$ on $J_{0}(X, A)$. Let $q_{1}, \cdots, q_{s}$ be as in the proof of 5.7. Since each $q$ is irreducible it has only simple roots. Thus $\operatorname{deg} q_{1} \cdots q_{s}=$ card $J_{0}(X, A)$ which we write as $n$. Since $q_{1} \cdots q_{s}$ is a monic polynomial in $A[z]$ we can find $w, t \in A[z]$ such that

$$
p=w\left(q_{1} \cdots q_{s}\right)+t, \quad \operatorname{deg} t<n
$$

by the division algorithm. Thus $t=p=f$ on $J_{0}(X, A)$ and then by the uniqueness of Lagrange interpolating polynomials $t=r$ and $r=f$ on $J_{0}(X, A)$.

We see from 5.7 and 5.8 that, under the hypotheses of 5.7 the question of approximability is effectively known once we know the finite set $J_{0}(X, A)$. The following shows that under these hypotheses the set $J_{0}(X, A)$ has another characterization.

Theorem 5.9. Let $X$ be a Lavrent'ev subset of $C$ with $d(X)<1$. Then

$$
J(X, A)=J_{0}(X, A)
$$

Proof. By 4.5, $J_{0}=J_{0}(X, A) \subset J(X, A)=J$, so we need only prove the reverse inclusion. Let $L$ be the imaginary quadratic field such that $A \subset I_{L}$. By $\S 2$ there is a positive integer $m$ such that $m I_{L} \subset A$. By 5.4 there is a monic $q \in I_{L}[z]$ such that $\|q\|<1$. Then for a sufficiently large positive integer $N, q_{0}=m q^{N}$ is a nonzero polynomial in $A[z]$ with $\left\|q_{0}\right\|<1$. This shows that $J$ is finite. Let $\alpha_{1}$ be any element of $J$. Then $q_{0}\left(\alpha_{1}\right)=0$ so $q\left(\alpha_{1}\right)=0$ and $\alpha_{1}$ is integral over $I_{L}$. Let $r \in I_{L}[z]$ be the minimal polynomial over $L$ of $\alpha_{1}$. We assume that $\alpha_{1} \notin J_{0}$, that is that not all the zeroes of $r$ lie in $X$ and 
infer a contradiction from this. Denote the zeroes of $r$ by $\left\{\alpha_{1}, \cdots, \alpha_{n}\right\}$ where

$$
\alpha_{i} \in X \quad \text { for } 1 \leqq i \leqq k
$$

and

$$
\alpha_{i} \notin X \quad \text { for } k<i \leqq n(k<n) .
$$

By 7.1 there is a $q_{1}^{\prime} \in I_{L}[z]$ such that

$$
\left|q_{1}^{\prime}\left(\alpha_{i}\right)-\frac{1}{2 m}\right|<\frac{1}{2 m} \quad \text { for } 1 \leqq i \leqq k .
$$

Then if $q_{1}=m q_{1}^{\prime}, q_{1} \in A[z]$ and

$$
\left|q_{1}\left(\alpha_{i}\right)-1 / 2\right|<1 / 2
$$

for $1 \leqq i \leqq k$.

Now, since $\alpha_{1} \in J$, if $q_{2}$ is any element of $A[z]$ with $\left\|q_{2}\right\|<1$, then $q_{2}\left(\alpha_{1}\right)=0$. The minimal polynomial $r$ of $\alpha_{1}$ then divides $q_{2}$ and so $q_{2}\left(\alpha_{i}\right)=0$ for $1 \leqq i \leqq n$. Thus $\left\{\alpha_{1}, \cdots, \alpha_{k}\right\}$ is contained in $J$. We write

$$
J=\left\{\alpha_{1}, \cdots, \alpha_{k}, \beta_{1}, \cdots, \beta_{l}\right\}
$$

where the $\beta_{j}$ 's are distinct and also distinct from the $\alpha_{i}^{\prime}$ 's. If $l=0$ define $q_{3}^{\prime}$ to be the polynomial 1. If $l>0$ define $q_{3}^{\prime}$ to be the product of the minimal polynomials over $L$ of the $\beta_{j}$ 's. Each $\beta_{j}$, being in $J$, is a zero of $q_{0}$ and therefore of $q$ and so is integral over $I_{L}$. Thus $q_{3}^{\prime}$ is an element of $I_{L}[z]$. Furthermore, $q_{3}=m M q_{3}^{\prime} \in A[z]$ and

$$
q_{3}\left(\beta_{i}\right)=0
$$

for $1 \leqq i \leqq l$.

Since no $\beta_{j}$ is conjugate to an $\alpha_{i}$

$$
q_{3}\left(\alpha_{i}\right) \neq 0 \text { for } 1 \leqq i \leqq n .
$$

By (1) and (3) there is a positive integer $s$ such that if

$$
q_{4}=q_{3} q_{1}^{s}
$$

then

$$
0<\left|q_{4}\left(\alpha_{i}\right)\right|<1
$$

for $1 \leqq i \leqq k$

Also, by (2), we have

$$
q_{4}\left(\beta_{i}\right)=0 \quad \text { for } 1 \leqq i \leqq l .
$$

Now it is easy to construct a continuous function $f$ with $\|f\|<1$ such that 


$$
f\left(\alpha_{i}\right)=q_{4}\left(\alpha_{i}\right)
$$

for $1 \leqq i \leqq k$

and

$$
f\left(\beta_{i}\right)=q_{4}\left(\beta_{i}\right)=0 \quad \text { for } 1 \leqq i \leqq l .
$$

This function $f$ is matchable on $J_{0}$ and by 5.8 , it is $A$-approximable on $X$. It is easy to see that we can choose $q_{5} \in A[z]$ with $\left\|f-q_{5}\right\|$ small enough to force $\left\|q_{5}\right\|<1$ and $\left|q_{5}\left(\alpha_{1}\right)\right|>0$ which is a contradiction.

In $\S 4$ we showed that if $d(X) \geqq 1$ then $A[z]$ is already uniformly closed in $C(X)$. The following is a partial converse to that result.

Proposition 5.10. Let $X$ be a Lavrent'ev subset of $C$ with $d(X)<1$. Then $A(z)$ is uniformly closed in $C(X)$ if and only if

$$
J(X, A)=X .
$$

In particular, if $X$ is infinite, then $A[z]$ is not uniformly closed in $C(X)$.

Proof. Suppose that $J=J(X, A)=X$ and that $f \in C(X)$. By 5.7 and 5.9 , if $f$ is $A$-approximable on $X$, it is $A$-matchable on $J=X$ and so $f \in A[z]$, which shows $A[z]$ is uniformly closed in $C(X)$.

On the other hand, if $J \neq X$, let $z_{0}$ be a point in $X$ but not in $J$. For any $y \in \boldsymbol{R}$ we can define a continuous function $f_{0}: J \cup\left\{z_{0}\right\} \rightarrow \boldsymbol{C}$ by $f_{0}(J)=\{0\}$ and $f_{0}\left(z_{0}\right)=y$. It is continuous where defined since $d(X)<1$ which implies that $J$ is finite, as in the proof of 5.7, so that the relative topology on $J \cup\left\{z_{0}\right\}$ is discrete. By Tietze's extension theorem there is a continuous extension $f$ of $f_{0}$ to all of $X$. But $f$ is obviously $A$-matchable on $J$ and so is $A$-approximable on $X$ by 5.7. Since $y$ is any real number this shows that there are uncountably many $A$-approximable $f$ in $C(X)$. On the other hand, $A[z]$ is countable, since $A$ is, so $A[z] \neq A[z]^{-}$, where the bar denotes uniform closure in $C(X)$.

The last statement now follows from the fact that $J(X, A)$ is finite whenever $d(X)<1$.

6. The real case. In this section we consider the problem analogous to that of $\S 5$ but where $X$ is a compact subset of $\boldsymbol{R}$. We take as our ring of coefficients any nonzero subring $R$ of $Z$. Such rings comprise the discrete nonzero subrings of $\boldsymbol{R}$. They are thus discrete subrings of $C$ but do not have rank 2 . The results follow readily from the corresponding results in the complex case.

To emphasize that $X \subset \boldsymbol{R}$. We use the symbol $x$ instead of $z$ to denote an arbitrary element of $X$. 
Note that in $\S 4$ the ring of coefficients $A$ was required to have rank 2 , hence the following definition is consistent with 4.4 .

Definition 6.1. For any compact subset $X$ of $\boldsymbol{R}$ let $J_{0}(X, Z)$ denote the union of the complete sets of conjugates integral over $\boldsymbol{Z}$ and entirely contained in $X$.

That is, $J_{0}(X, Z)$ is the union of the complete sets of conjugate algebraic integers contained in $X$.

We now proceed to show that this separate definition is, in a sense, unnecessary.

Proposition 6.2. Let $X$ be a compact subset of $\boldsymbol{R}$. Then for any imaginary quadratic field $L$, we have

$$
J(X, Z)=J\left(X, I_{L}\right) .
$$

Proof. Since $B(X, Z) \subset B\left(X, I_{L}\right)$ the inclusion $J\left(X, I_{L}\right) \subset J(X, Z)$ is obvious. On the other hand, let $x_{0} \in J(X, Z)$ and let $p \in B\left(X, I_{L}\right)$. Then $\|p\|<1$, so for some positive integer $n$,

$$
\left\|p^{n}\right\|=\|p\|^{n}<1 / 2 \text {. }
$$

Then we have

$$
\left\|\operatorname{Re}\left(p^{n}\right)\right\|<1 / 2 \text { and }\left\|\operatorname{Im}\left(p^{n}\right)\right\|<1 / 2 \text {. }
$$

Furthermore, from $\S 2$ we see that $2 \operatorname{Re}\left(p^{n}\right)$ and $(2 / \sqrt{|d|}) \operatorname{Im}\left(p^{n}\right)$ are in $Z[x]$, where $L=Q(\sqrt{d})$ with $d$ a square free integer and $\operatorname{Re}\left(p^{n}\right)$ (resp. Im $\left(p^{n}\right)$ ) denotes the polynomial obtained by replacing the coefficients of $p^{n}$ by their real (resp. imaginary) parts. Also

$$
\left\|2 \operatorname{Re}\left(p^{n}\right)\right\|=2\left\|\operatorname{Re}\left(p^{n}\right)\right\|<1
$$

and

$$
\left\|(2 / \sqrt{|d|}) \operatorname{Im}\left(p^{n}\right)\right\|<(1 /|d|)^{1 / 2} \leqq 1
$$

and so by definition of $J(X, Z)$

$$
2 \operatorname{Re}\left(p^{n}\right)\left(x_{0}\right)=0
$$

and

$$
(2 / \sqrt{|d|}) \operatorname{Im}\left(p^{n}\right)\left(x_{0}\right)=0 .
$$

But $p^{n}\left(x_{0}\right)=\left(\operatorname{Re}\left(p^{n}\right)\right)\left(x_{0}\right)+i\left(\operatorname{Im}\left(p^{n}\right)\right)\left(x_{0}\right)$ and so $p^{n}\left(x_{0}\right)=0$, which implies that $p\left(x_{0}\right)=0$. Hence $x_{0} \in J\left(X, I_{L}\right)$ and $J(X, Z) \subset J\left(X, I_{L}\right)$.

Before proving the next proposition we note that if $X$ is a compact subset of $\boldsymbol{R}$ it is Lavrent'ev by 5.2 or by the Stone-Weierstrass theorem. 
Proposition 6.3. Let $X$ be a compact subset of $\boldsymbol{R}$ with $d(X)<1$. Then for any imaginary quadratic field $L$,

$$
J_{0}(X, Z)=J_{0}\left(X, I_{L}\right) \text {. }
$$

Proof. If $x_{0} \in J_{0}(X, Z)$, then $x_{0}$ is a root of a monic polynomial $p \in Z[x]$ which has all of its roots in $X$. Thus $z_{0}$ is integral over $I_{L}$. The minimal polynomial $q$ of $x_{0}$ over $L$ is then an element of $I_{L}[z]$, monic, irreducible, and divides $p$ so that the roots of $q$ all lie in $X$. Thus $x_{0} \in J_{0}\left(X, I_{L}\right)$.

For the reverse inclusion notice that $J_{0}\left(X, I_{L}\right)=J\left(X, I_{L}\right)=J(X, Z)$ by 6.2 and 5.9. This shows, in particular, that $J_{0}\left(X, I_{L}\right)$ is independent of the choice of $L$. Suppose then, that $L=\boldsymbol{Q}(i)$ where $i^{2}=-1$. Then $I_{L}$ is the set of Gaussian integers. If $x_{0} \in J_{0}\left(X, I_{L}\right)$ then it is a root of monic, irreducible $p$ in $I_{L}[z]$ having all of its roots in $X$. Then the coefficients of $p$, being simply the elementary symmetric polynomials in the roots, are in $I_{L} \cap \boldsymbol{R}$. But $I_{L} \cap \boldsymbol{R}=\boldsymbol{Z}$, so $x_{0} \in J_{0}(X, Z)$. Since $x_{0}$ is any element of $J_{0}\left(X, I_{L}\right), J_{0}\left(X, I_{L}\right) \subset J_{0}(X, Z)$.

THeOREM 6.4. If $X$ is any compact subset of the real line $\boldsymbol{R}$ with $d(X)<1$, then

$$
J(X, Z)=J_{0}(X, Z) .
$$

Proof. This is immediate from 6.2, 6.3 and 5.9.

A natural question at this point is whether or not the hypothesis $d(X)<1$ can be dropped from 6.4 or 5.10 . We see that it cannot be dropped in either case by the following argument.

Let $L$ be an imaginary quadratic field and $X$ any uncountable compact subset of $C$ with $d(X) \geqq 1$. Then we know that $B\left(X, I_{L}\right)=\{0\}$ by the comments following 4.2 and so $B(X, Z)=\{0\}$. This implies that $J\left(X, I_{L}\right)=J(X, Z)=X$ by definition. On the other hand, every element of $J_{0}(X, Z)$ (respectively $J_{0}\left(X, I_{L}\right)$ ) is algebraic over $\boldsymbol{Q}$ and so $J_{0}(x, \boldsymbol{Z})$ (respectively $J_{0}\left(x, I_{L}\right)$ ) is countable and so not equal to $X=$ $J(X, Z)=J\left(X, I_{L}\right)$.

Another question is whether or not it is necessary to consider polynomials with complex coefficients when seeking the Chebyshev polynomials $t_{n}=t_{n}(x, X)$ for $X \subset \boldsymbol{R}$. This is not necessary since the Chebyshev polynomials have real coefficients in this case since

$$
\left\|\operatorname{Re}\left(t_{n}\right)\right\| \leqq\left\|t_{n}\right\| \text {. }
$$

Theorem 6.5. If $X$ is a compact subset of $\boldsymbol{R}$ and $R$ is a nonzero subring of $\boldsymbol{Z}$, then a function $f$ in $C^{r}(X)$ is $R$-approximable if and only if $f$ is $R$-matchable on $J(X, Z)$. 
Proof. If $f$ is $R$-approximable then it is $R$-matchable on $J(X, Z)$ by 4.6 and 6.2. Conversely, suppose that $f$ is $R$-matchable on $J(X, Z)$. If $d(X) \geqq 1$ then $B(X, Z)=\{0\}$ by the comments following 4.2 and so $J(X, \boldsymbol{Z})=X$. Thus $f$ is in fact, a member of $R[x]$. If $d(X)<1$ assume that $f$ is $R$-matchable on $J(X, Z)$, say by $p \in R[x]$. Since $R$ is a nonzero subring of $\boldsymbol{Z}$ we have $R=n \boldsymbol{Z}$ for some positive integer $n$. It suffices to approximate $q=f-p$. In fact it suffices to approximate $q_{0}=q / n$ by an element of $Z[x]$, since if

$$
|q / n-p|<\varepsilon / n
$$

then

$$
|q-n q|<\varepsilon
$$

and $n p \in(n Z)[x]=R[x]$. Let $L$ be any imaginary quadratic field (the Gaussian numbers, for example). Since $q_{0}$ is zero on $J(X, Z)=J\left(X, I_{L}\right)$ it is $I_{L}$-matchable on $J\left(X, I_{L}\right)$. Thus, for any $\varepsilon>0$, there exists a $p \in I_{L}[z]$ such that

$$
\left\|p-q_{0}\right\|_{X}<\varepsilon / 2,
$$

by 5.7 and 5.9. Then for any $x \in X$

$$
\begin{aligned}
\frac{\varepsilon}{2} & >\mid \operatorname{Im}(p(x)-f(x)): \\
& =|\operatorname{Im}(p(x))| \\
& =|(\operatorname{Im} p)(x)| .
\end{aligned}
$$

Since $p=\operatorname{Re} p+i \operatorname{Im} p$

$$
\|p-\operatorname{Re} p\|<\varepsilon / 2,
$$

and by (1) we have

$$
\left\|\operatorname{Re} p-q_{0}\right\|_{X}<\varepsilon .
$$

From the above proof we have the following

CoRollary 6.6. If $d(X) \geqq 1, f$ is $R$-approximable on $X$ if and only if $f$ is already an element of $R[x]$.

Since the transfinite diameter of an interval is one fourth its length we have the following.

Corollary 6.7. If $X=[a, b]$ and $b-a \geqq 4$ then $f$ is $R$-approximable on $X$ if and only if $f$ is already an element of $R[x]$. 
Using essentially the same arguments as for their respective counterparts 5.8 and 5.10, the following can be proved.

Proposition 6.8. Let $X$ be a compact subset of $\boldsymbol{R}$ with $d(X)<1$ and $R$ a nonzero subring of $Z$. Then an element $f$ of $C^{r}(X)$ is $R$ approximable on $X$ if and only if the Lagrange interpolating polynomial for $f$ on $J(X, Z)$ is an element of $R[x]$.

Proposition 6.9. Let $X$ be a compact subset of $\boldsymbol{R}$ with $d(X)<1$ and $R$ a nonzero subring of $Z$. Then $R[z]$ is uniformly closed in $C^{r}(X)$ if and only if

$$
J(X, Z)=X,
$$

In particular, if $X$ is infinite, then $Z[x]$ is not uniformly closed in $C^{r}(X)$.

The main result of this section is Theorem 6.5. It reduces the problem to that of finding $J(X, Z)$. For some nontrivial cases where $J(X, Z)$ has been determined see $[7, \S 5]$.

7. Appendix. An approximation theorem. Throughout this section let $A$ be any discrete subring of $C$ of rank 2 and containing the identity. Let $L$ be the unique imaginary quadratic field such that $A \subset I_{L}$.

THEOREM 7.1. Let $\alpha_{1}, \cdots, \alpha_{n}$ be a complete set of conjugates over $L, \varepsilon$ any position number, and $z_{2}, \cdots, z_{n}$ any complex numbers. Then there is a polynomial $q \in A[z]$ such that

$$
\left|q\left(\alpha_{i}\right)-z_{j}\right|<\varepsilon \quad \text { for } 2 \leqq j \leqq n .
$$

Proof. This is a consequence of the "very strong approximation theorem," c.f. [10, p. 77, 33: 11].

THEOREM 7.2. Let

$$
\begin{aligned}
& \alpha_{11}, \cdots, \alpha_{1 r_{1}} \\
& \alpha_{21}, \cdots, \alpha_{2 r_{2}} \\
& \cdots \cdots, \cdots \\
& \alpha_{s 1}, \cdots, \alpha_{s r_{s}}
\end{aligned}
$$

be an array (not necessarily rectangular) with each row an incomplete set of conjugates over $L$ and where the minimal polynomials $p_{1}, \cdots, p_{s}$ in $L[z]$ satisfied by the respective rows are distinct. Then if the 
array

$$
\begin{gathered}
z_{11}, \cdots, z_{1 r_{1}} \\
z_{21}, \cdots, z_{2 r_{2}} \\
\ldots \cdots \cdots \\
z_{s 1}, \cdots, z_{s r_{s}}
\end{gathered}
$$

consists of any complex numbers and $\varepsilon>0$, there exists a $q \in A[z]$ such that

$$
\left|q\left(\alpha_{i j}\right)-z_{i j}\right|<\varepsilon \quad \text { for } 1 \leqq j \leqq r_{i}, 1 \leqq i \leqq s
$$

Proof. Let

$$
q_{i}^{\prime}=\left(\prod_{k=1}^{s} p_{k}\right) / p_{i}
$$

for $1 \leqq i \leqq s$

Then $q_{i}^{\prime}\left(\alpha_{k j}\right)=0$ if and only if $k \neq i$ by definition of the $p$ 's. Furthermore, each $q_{i}^{\prime}$ is a polynomial with coefficients in $L$. But $L$ is the field of quotients of $A$ and we can suppose that each coefficient of each $q_{i}^{\prime}$ appears as a ratio of elements of $A$. If $k_{i}$ is the product of the denominators of the coefficients of $q_{i}^{\prime}$ then the coefficients of the polynomial $k_{i} q_{i}^{\prime}$ lie in $A$. For $1 \leqq i \leqq s$, by 7.1 , there is a $q_{i}^{\prime \prime} \in A[z]$, such that

$$
\left|q_{i}^{\prime \prime}\left(\alpha_{i j}\right)-\frac{z_{i j}}{k_{i} q_{i}^{\prime}\left(\alpha_{i j}\right)}\right|<\frac{\varepsilon}{\left|k_{i} q_{i}^{\prime}\left(\alpha_{i j}\right)\right|} \quad \text { for } 1 \leqq j \leqq r_{i}
$$

Thus

$$
\left|\left(q_{i}^{\prime \prime} k_{i} q_{i}^{\prime}\right)\left(\alpha_{i j}\right)-z_{i j}\right|<\varepsilon \quad \text { for } 1 \leqq j \leqq r_{i} \text {. }
$$

If we set $q=q_{1}^{\prime \prime} k_{1} q_{1}^{\prime}+\cdots+q_{s}^{\prime \prime} k_{s} q_{s}^{\prime}$, then $q$ is in $A[z]$ and $q\left(\alpha_{i j}\right)=$ $\left(q_{i}^{\prime \prime} k_{i} q_{i}^{\prime}\right)\left(\alpha_{i j}\right)$ since $q_{k}^{\prime}\left(\alpha_{i j}\right)=0$ if $k \neq i$. Thus

$$
\left|q\left(\alpha_{i j}\right)-z_{i j}\right|=\left|\left(q_{i}^{\prime \prime} k_{i} q_{i}^{\prime}\right)\left(\alpha_{i j}\right)-z_{i j}\right|<\varepsilon
$$

for all $i, j$.

We note that another way of looking at Theorem 7.2 is the following.

COROLlary 7.3. If $\left\{\alpha_{1}, \cdots, \alpha_{k}\right\}$ is any set of algebraic numbers which does not contain a complete set of conjugates over $L$, then the set of k-tuples

$$
\left\{\left(p\left(\alpha_{1}\right), \cdots, p\left(\alpha_{k}\right)\right): p \in A[z]\right\}
$$

is dense in $\boldsymbol{C}^{k}$. 
I wish to thank Professor Edwin Hewitt for his help and encouragement as thesis advisor. The appendix and its application herein arose out of conversations with Professor David Cantor.

\section{REFERENCES}

1. N. I. Achieser, Theory of Approximation, Frederick Ungar, New York, 1956.

2. N. Bourbaki, Éléments de mathématique, Hermann et Cie, Paris.

3. —_ Éléments de mathématique, V, Première partie, Les structures fondamentales de l'analyse, Livre III, Topologie générale, Chap. V-VIII, Actualités Sci. et Ind. 1235, Hermann et Cie., 1963.

4. M. Fekete, Approximations par polynomes avec conditions diophantienes, I et II, C. R. Acad. Sci. Paris 239 (1954), 1337-1339 and 1455-1457.

5. L. B. O. Ferguson, Uniform approximations by polynomials with coefficients in discrete subrings of $\boldsymbol{C}$, Dissertation, University of Washington, 1965.

6. E. Hewitt and K. A. Ross, Abstract Harmonic Analysis, Volume 1, Springer-Verlag, Berlin-Göttingen-Heidelberg, 1963.

7. E. Hewitt and H. S. Zuckerman, Approximation by polynomials with integral coefficients, a reformulation of the Stone-Weierstrass theorem, Duke Math. J. 26 (1959), 305-324.

8. E. Hille, Analytic Function Theory, Volume II, Ginn and Co., New York, 1962.

9. M. A. Lavrent'ev, Sur les functions d'une variable complex représentables par des séries de polynomes, Actualités Sci. et Ind. 441, Hermann et Cie., Paris, 1936.

10. O. T. O'Meara, Introduction to Quadratic Forms, Springer-Verlag, Berlin-GöttingenHeidleberg, 1963.

11. G. Pólya und G. Szego, Aufgaben und lehrsätze aus der analysis, Zweiter band, Zweite auflage, Die grundlehren der mathematischen wissenschaften in einzeldarstellungen, Band XX, Springer-Verlag, Berlin-Göttingen-Heidelberg, 1954.

12. T. J. Rivlin and H. S. Shapiro, Some uniqueness problems in approximation theory, Comm. Pure Appl. Math. 13 (1960), 35-47.

13. E. Weiss, Algebraic Number Theory, International Series in Pure and Applied Mathematics, McGraw-Hill Book Co., Inc., 1963.

Received September 27, 1966, and in revised form August 16, 1967. This work is an improvement on a major part of the author's thesis [5] performed under Professor Edwin Hewitt.

UNIVERSITY OF WASHINGTON

University of California, Riverside 



\section{PACIFIC JOURNAL OF MATHEMATICS}

EDITORS

H. ROYDEN

Stanford University

Stanford, California

R. R. Phelps

University of Washington

Seattle, Washington 98105
J. DugundJI

Department of Mathematics

University of Southern California

Los Angeles, California 90007

\section{RICHARD ARENS}

University of California

Los Angeles, California 90024

\section{ASSOCIATE EDITORS}
E. F. BeCKENBACH
B. H. NeUmanN
F. WOLF
K. YOSIDA

\section{SUPPORTING INSTITUTIONS}

UNIVERSITY OF BRITISH COLUMBIA CALIFORNIA INSTITUTE OF TECHNOLOGY UNIVERSITY OF CALIFORNIA MONTANA STATE UNIVERSITY UNIVERSITY OF NEVADA NEW MEXICO STATE UNIVERSITY OREGON STATE UNIVERSITY UNIVERSITY OF OREGON OSAKA UNIVERSITY UNIVERSITY OF SOUTHERN CALIFORNIA
STANFORD UNIVERSITY UNIVERSITY OF TOKYO UNIVERSITY OF UTAH WASHINGTON STATE UNIVERSITY UNIVERSITY OF WASHINGTON

AMERICAN MATHEMATICAL SOCIETY CHEVRON RESEARCH CORPORATION TRW SYSTEMS NAVAL WEAPONS CENTER 


\section{Pacific Journal of Mathematics \\ Vol. 27, No. $1 \quad$ January, 1968}

Willard Ellis Baxter, On rings with proper involution ............... 1

Donald John Charles Bures, Tensor products of $W^{*}$-algebras........... 13

James Calvert, Integral inequalities involving second order derivatives . . . . 39

Edward Dewey Davis, Further remarks on ideals of the principal class.... 49

Le Baron O. Ferguson, Uniform approximation by polynomials with integral

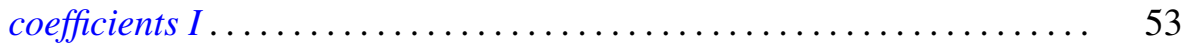

Francis James Flanigan, Algebraic geography: Varieties of structure

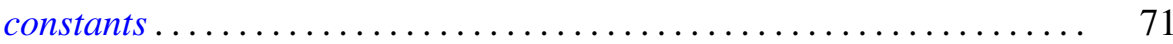

Denis Ragan Floyd, On QF -1 algebras ..................... 81

David Scott Geiger, Closed systems of functions and predicates ......... 95

Delma Joseph Hebert, Jr. and Howard E. Lacey, On supports of regular Borel measures ................................... 101

Martin Edward Price, On the variation of the Bernstein polynomials of a function of unbounded variation ........................ 119

Louise Arakelian Raphael, On a characterization of infinite complex matrices mapping the space of analytic sequences into itself........ 123

Louis Jackson Ratliff, Jr., A characterization of analytically unramified

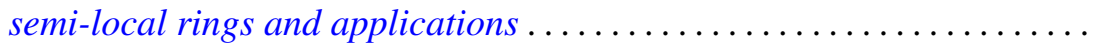

S. A. E. Sherif, A Tauberian relation between the Borel and the Lototsky transforms of series ................................ 145

Robert C. Sine, Geometric theory of a single Markov operator .......... 155

Armond E. Spencer, Maximal nonnormal chains in finite groups......... 167

Li Pi Su, Algebraic properties of certain rings of continuous functions .... 175

G. P. Szegô, A theorem of Rolle's type in $E^{n}$ for functions of the class $C^{1} \ldots 193$

Giovanni Viglino, A co-topological application to minimal spaces ........ 197

B. R. Wenner, Dimension on boundaries of $\varepsilon$-spheres ............... 201 